\title{
NOVA DELPHINI 1967: RADIAL VELOCITIES AND EXCITATION POTENTIALS OF LINES
}

\author{
E. SZUMIEJKO and T. JARZĘBOWSKI \\ Wroclaw Observatory, Poland \\ and
}

E. BQ ARTL

Tautenburg Observatory, G.D.R.

\begin{abstract}
Radial velocities were derived from absorption lines of various excitation potentials; there seems to exist a small dependence on this potential.
\end{abstract}

We have investigated the measured radial velocities of Nova Del 1967 (HR Del) and the relationship between the radial velocities and the excitation potentials of the lines.

The study has been based on the spectroscopic material obtained with the $2 \mathrm{~m}$-universal telescope of the Tautenburg Observatory. The plates of $30 \AA \mathrm{mm}^{-1}$ dispersion were taken using the Cassegrain-grating-spectrograph in 1967 and 1968.

The measurements of radial velocities have been made from direct measurements of the plates and, independently, from the microphotometer tracings (in this case the

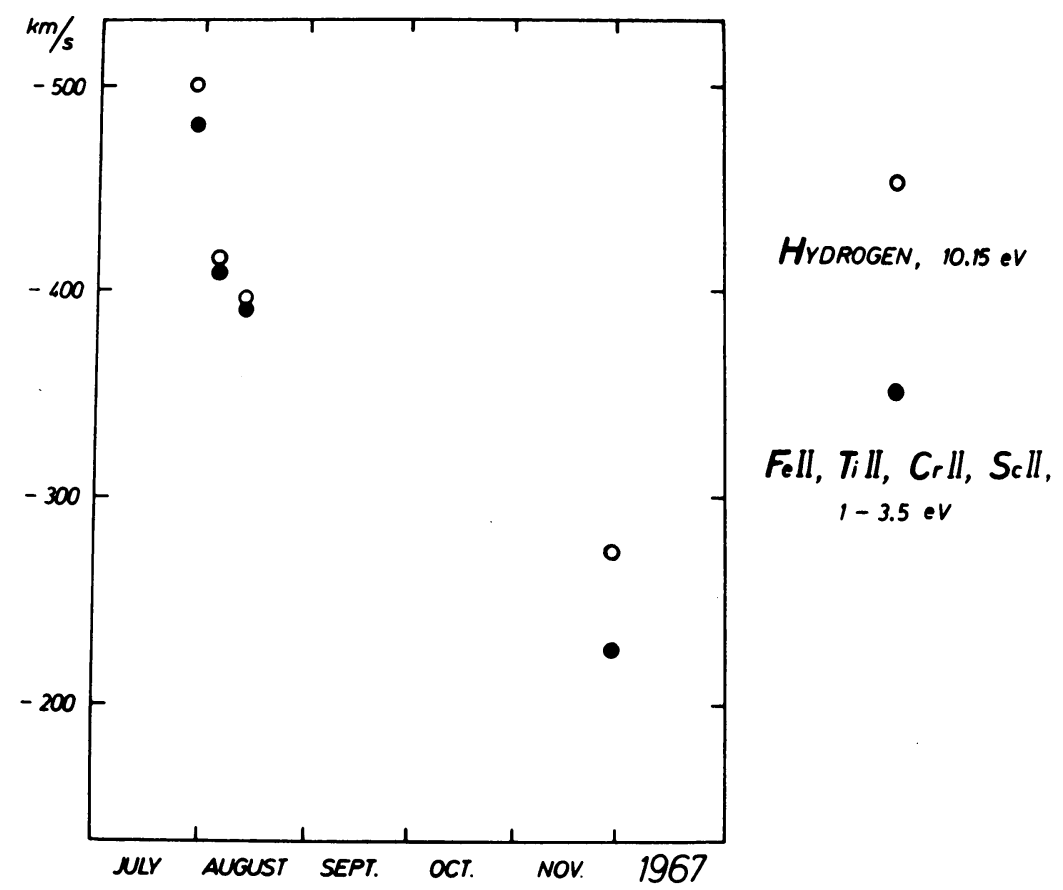

Fig. 1. Radial velocities from the absorption lines of hydrogen (open circles), and from the absorp tion lines of metals (dots). 
comparison spectrum was superimposed on the spectrum of the star). The accuracy of the measurements - for sharp and unblended lines - was of the order of $0.1 \AA$ which is equivalent to $\pm 6 \mathrm{~km} \mathrm{~s}^{-1}$.

The results obtained from the study of the absorption lines of four plates (29 July, 5 and 8 August, and 29 November 1967) are shown in Figure 1. The velocities obtained from hydrogen lines (excitation potential $10.15 \mathrm{eV}$ ) are higher than the velocities obtained from the lines of metals (excitation potentials $1-3.5 \mathrm{eV}$ ).

In Figure 2 the absorption line radial velocities for July 29 are plotted against the corresponding values of the excitation potential. The increase of velocity with increase of excitation potential is rather evident.

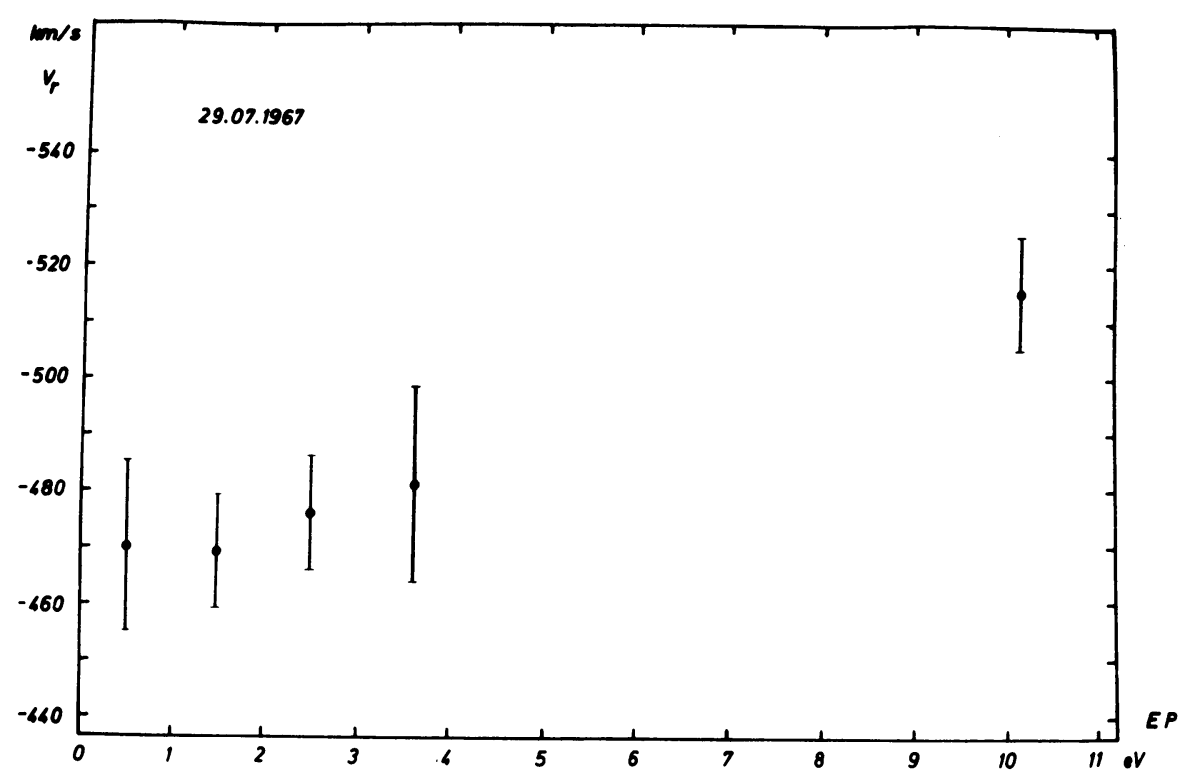

Fig. 2. Velocities vs. excitation potential on 1967 , July 29. Mean values were taken for lines of similar excitation potential. Standard errors are indicated by vertical bars.

If the discussed relationship is real, it could indicate that the lines of higher excitation potential originate in deeper layers, where the velocity of expansion is greater.

The details of this investigation, as well as the results obtained from the spectroscopic material of 1968, will be published in Acta Astronomica in a paper by Bartl, Jarzębowski, and Szumiejko. 